\title{
Revisión de las estrategias usadas en la gestión pública durante la pandemia del covid-19
}

\author{
César Romero Silva \\ cesarmarcosromeros2020@gmail.com \\ Fernando Emilio Escudero Vílchez \\ fescudero@silfersystem.com \\ Silvia Liliana Salazar Llerena \\ Ssalazar@silfersystem.com \\ Universidad César Vallejo \\ Lima-Perú
}

\section{RESUMEN}

OBJETIVO: La presente investigación tuvo como propósito principal de revisar las estrategias que se optaron por realizar tras la pandemia del COVID-19 que atormento al mundo el pasado 2020. Una estrategia es un plan de acción que realizan una o grupo de personas de largo o corto plazo para así poder cumplir tanto metas como objetivos propuestos, estas estrategias son de vital importancia por si llegan a suceder situaciones de gravedad como la actual pandemia, por lo cual se va a ser de vital importancia la revisión de distintos autores sobre el tema a investigar.METODOLOGíA: La metodología empleada dentro del presente artículo ha sido bajo un enfoque cuantitativo, mediante la revisión de variada bibliografía de organismos nacionales e internacionales como también autores referentes a la información previamente investigada por ellos. RESULTADOS: Como resultado se evidenció que una eficiente gestión pública permite llevar a cabo planes, estrategias y políticas nacionales que estén dirigidas a satisfacer las necesidades que presenta la ciudadanía. CONCLUSIÓN: Finalmente, se concluye que, la gestión pública de muchos países ha actuado de manera rápida para poder proteger a sus ciudadanos, muchas si no todas se han dado cuenta de lo inestable que puede llegar a ser el sistema de salud y lo no preparado que estaban los gobiernos para poder responder ante una situación crítica como esa.

Palabras clave: gestión pública; covid-19; sector público 


\title{
Review of the strategies used in public management during the covid pandemic-19
}

\begin{abstract}
OBJECTIVE: The main purpose of this research was to review the strategies that were chosen to be carried out after the COVID-19 pandemic that tormented the world in 2020. A strategy is an action plan carried out by one or group of people long or short-term in order to meet both proposed goals and objectives, these strategies are of vital importance in case serious situations such as the current pandemic happen, for which the review of different authors on the subject will be of vital importance. Investigate. METHODOLOGY: The methodology used in this article has been under a quantitative approach, through the review of various bibliography of national and international organizations as well as authors referring to the information previously investigated by them. RESULTS: As a result, it was evidenced that efficient public management allows carrying out national plans, strategies and policies that are aimed at satisfying the needs of citizens. CONCLUSION: Finally, it is concluded that the public management of many countries has acted quickly to protect their citizens, many if not all have realized how unstable the health system can be and how unprepared that governments were there to respond to a critical situation like that.
\end{abstract}

Keywords: public management; covid-19; public sector

Artículo recibido: 30 noviembre. 2021 Aceptado para publicación: 29 diciembre 2021 Correspondencia: cesarmarcosromeros2020@gmail.com

Conflictos de Interés: Ninguna que declarar 


\section{INTRODUCCIÓN}

La gestión pública es el órgano que administra una empresa o estado de gran relevancia, buscando brindar y mejorar sus servicios a las personas. Es por ello que dentro de la gestión pública se observa las variedades de estrategias y herramientas que implementa para mejorar la efectividad y eficacia de los servicios que ofrecen, mejorando la gestión y proceso de trámites, el empleo de tecnologías con el objetivo de realizar las áreas de trabajo con mayor efectividad, y además mejorar las capacidades de los colaboradores mediante las tecnologías, mejorando la calidad que se brinda por lo que es importante recalcar si la gestión pública tuviera una mala organización y/o administración, ya sea para cualquiera de sus distintos sectores, la efectividad y eficiencia de estos, sería pésima y se tendría un retraso con los objetivos que se la población esperaba (Machín et al., 2019).

Actualmente, la gestión pública es considerada por parte del Estado como un proceso que tiene como propósito principal satisfacer las carencias e ilusiones que puedan presentar los ciudadanos, todo esto por medio de la instauración de herramientas de temple más transparente y eficiente. Además, este es un proceso que se da manera gradual y solo en los gobiernos que cuenten con objetivos previamente establecidos, asimismo, es necesario realizar un seguimiento para garantizar que se esté cumpliendo en su totalidad (Cepeda \& Cifuentes, 2019).

La gestión pública nace como una alternativa para los gobiernos que ambicionan reforzar a la democracia representativa, debido a que en esta se refleja que existe una gran carencia en el marco de la aceptación por parte de los ciudadanos; es decir, se desconfía de los

gobiernos. Del mismo modo menciona que la gestión pública en América Latina es importante aplicarla debido a sus grandes beneficios que se define como una agenda que involucra cambios en diferentes ámbitos de gestión pública, los que abarca los diversos sistemas de planeación y los mecanismos de gestión (Pliscoff, 2017). En la misma linea,los autores Hodges y Howieson et al. (2017) mencionan que debido a los cambios que han estado experimentado los cambios sociales, políticos y económicos se debe implementar de manera optima las herramientas tecnologicas a fin de poder conseguir la participaciòn de la ciudadania.

También se define a la gestión pública como un mecanismo empleado por los gobiernos con el propósito de manifestar una noción a la población de la existencia de un debido 
reforzamiento a la democracia representativa, esto debido a que existe un alto grado de desconfianza por parte de los ciudadanos con los gobiernos. El Estado es el ente principal de supervisar y administrar eficazmente las actividades o procesos que se realizan en pro de la población pública, la mejor forma de hacerlo es incentivando la participación ciudadana y consecuentemente brindarles la garantía de que sus opiniones y decisiones son de vital importancia al momento de tomar una decisión (Morveli, 2021).

Por otro lado, la gestión de salud pública se ha visto muy afectada debido a los cambios ocurridos por la pandemia del COVID-19 ya que requiere diversas estrategias y prevención de que la ciudadanía se pueda adaptar al impacto de la pandemia. Además, los cambios sociales y económicos provocados por la pandemia, junto con las tradicionales dificultades de acceso y adherencia al tratamiento, ciertamente se agravarán durante este período y, por lo tanto, agravarán su condición (Ornell et al.,2020).

Llerena y Sánchez (2020) se debió tener que optar como estrategia extrema el reclutamiento social para así evitar contraer más contagios, como otras medidas dentro de todo el sistema social, político, económico, cultural y organizacional que condujo a un incierto de problemáticas a los ciudadanos por no estar preparados para un acontecimiento de tal magnitud.

Además, Bansal et al. (2020) señalaron que debido a la pandemia del COVID-19 se tuvo que implementar nuevas estrategias para poder implementar la gestion publica a favor de los ciudadanos debido a todo lo que estaba ocurriendo en nuestro pais,lo que afecto al sector salud,educativo,economia,etc.

\section{ESTRATEGIAS METODOLÓGICAS O MATERIALES Y MÉTODOS}

La presente investigación se realizó con un enfoque cuantitativo,de acuerdo con Snyder (2019), señalo que una investigación cuantitativa se basa en la observación que se adapta de manera efectiva en el trabajo, además permitiendo recolectar datos y procesarlos con el objetivo de determinar las hipótesis planteadas.

En la misma linea Tobi y Kampen (2018) señalan que esta permitió evidenciar los resultados de manera numérica y cuantificar los productos en aspectos estadísticos, asimismo recopilar información a manera de verificar o corroborar las hipótesis y/o teorías planteadas. 


\section{TÉCNICAS E INSTRUMENTOS DE RECOLECCIÓN DE DATOS}

Por otro lado, los datos utilizados se han originado a partir de una relación hipotéticadeductiva, la que nos permitirá verificar la credibilidad de los mismos, siendo que la verificación será de manera empírica. Por ende, se refiere a las variables para poder llegar a una conclusión en este caso sería la hipótesis para verificar su credibilidad y veracidad, y en caso el resultado fuera optimo se concluiría que hay una relación con la teoría inicial y los planteamientos del problema de la investigación (Sánchez,2019).

Así también, los datos utilizados se han originado a partir de una relación hipotéticadeductiva, siendo así que la verificación será de manera empírica y las variables han sido estudiadas por la relación entre ellas, esto con la finalidad de obtener un mayor entendimiento y comprensión de las mismas en conjunto, así como de su importancia de investigación (Núñez,2017).

\section{Procedimiento}

Se realizó la búsqueda bibliográfica y sistemática de artículos científicos en las siguientes bases de datos: Redalyc, Scielo y Scopus. La búsqueda y selección de los artículos inició en el período 2017 hasta el año 2021, para la investigación se decidió la selección de los artículos bajo una estructura relacionada con las variables, que consistió en realizar una búsqueda de información enfocada en el uso de las estrategias y la relación con la gestión pública en el Perú, sus características, normativa nacional, beneficios, enfocándonos en la incidencia de una variable sobre la otra.

Es por ello que, los datos obtenidos tienen el objetivo de ser desarrollados de manera correcta en el presente trabajo, dado que son parte de una revisión bibliográfica ardua relacionada a la temática mencionada anteriormente, los criterios para su selección fueron la fecha de publicación, el contenido veraz y verificable de la información, así es como se desarrollan; esta será utilizada como sustento estadístico en el análisis que se llevará a cabo.

\section{RESULTADOS Y DISCUSIÓN}

\section{Resultados}

Estas iniciativas significaron un alivio y esperanza para los pobladores, que comenzaro a sentir que al fin podrian salir poco a poco del estado deplorable en el que muchos se econtraban. Pero lamentablemente estos planes tuvieron ciertos margenes de error, donde 
muchas familias fueron estafadas por personas que "les facilitaban el tramite de retiro de dinero" o el dinero iba dirigido a familias que no tenian urgencias economicas.

En el apartado de salud se implementaron mayor cantidad de pruebas, estableciendo cuatro tipos: Pruebas rápidas, moleculares, antígenas y serologicas, teniendo alrededor de 65000 pruebas diarias, abarcando de mejor manera la identificación de contagiados. Actualmente se establecieron planes de vacunacion, dirgidos a la población de riesgo y trabajadores de primera linea, llegando a un total de 933000 ciudadanos vacunados, en los que se encuentran adultos mayores, personal de fuerzas armadas y personal médico. Es así como podemos encontrar muchas amenazas a las ideas planteadas en el marco de reactivación economica y prevención de contagio, los cuales se vieron frustrados por la mala gestion de planes de contingencia y la falta de colaboracion por parte de los ciudadanos.

\section{Discusiòn}

Cohen et al. (2017) refieren que implementar puede impulsan el interés de los ciudadanos en la toma de decisiones por parte de las autoridades, lo que a su vez los motivaría potencialmente a tener una participación más activa en los asuntos públicos. Por ende, se pudo evidenciar que hay una relación colaborativa entre las TIC que es un medio para informar a la ciudadanía de una manera más sencilla y colaborativa para incentivar su participación democrática.

McBride y Draheim (2020) afirman que debe existir una relación entre la gestión pública y estrategias innovadora a través de las herramientas tecnológicas con el fin de integrar una dinámica que favorezca a la ciudadanía y cumpla las expectativos que estos presentan sobre la gestión que se realiza.

Mauro y Giancotti (2021) mencionan que debido a la pandemia del COVID-19 se tuvo que implementar nuevos cambios y estrategias por parte del gobierno durante el 2020 hasta la actualidad. Ademas se menciona que el hecho de implementar nuevas ideas para mejorar o que sea mas eficiente la gestiòn pùblica por lo que introdujeron el gerencialismo, la regionalización y los cuasimercados en el Sistema Nacional de Salud. Como resultado, se obtuvo que la promulgación de nuevos decretos, el gobierno central intervino directamente en la gestión del sistema de salud mediante la introducción de medidas específicas destinadas a aumentar el número de camas y personal hospitalario, que antes se reducía. 
Hellewell et al. (2020) refieren que debido a los cambios ocurridos por la pandemia del COVID-19 se ha tenido que implementar diversas estrategias por parte de las autoridades para poder brindar soluciones a corto plazo a favor de los ciudadanos y tratar de manejar la situaciòn en el pais.Ademas se pudo implementar nuevas estrategias en la gestiòn pùblica para que se pueda tomar en consideraciòn la participaciòn de los ciudadanos.

Troy (2020) menciona que las herramientas digitales son de gran ayuda para la comunicación o la interacción entre los ciudadanos y el gobierno, el internet ha alterado la vida de las personas, inclusive en la gobernanza se han realizado cambios para mejorar la gestión y la administración de los recursos, todo esto con el objetivo de trascender la política y generar un mayor alcance.

Rana et al. (2017) también precisó que la gestión que se basa en una gobernanza electrónica permite que la política tenga un reforzamiento ético en la labor de los funcionarios, asimismo, permite reducir los costos y los tiempos. Cabe señalar que no se trata de trasladar la participación de los ciudadanos al área virtual, sino de amplificar esta participación con un manejo basado en la comunicación y las críticas constructivas para que el gobierno pueda dictar medidas y actividades que beneficien a la comunidad en general.

Oluyase et al. (2021) mencionan a consecuencia de la trasformacion del sector de salud,las autoridades no cumplieron sus funciones de forma optima,debido a la falta de informacion brindada a la ciudadania,el gobierno tampoco fue eficiente en el proceso que atravesaron los paises a nivel mundial debido a los contagios masivos provocados por la COVID-19. Por otro lado,mencionaron que eso mejoro en el año 2021 debido a nuevas estrategias implementadas en la gestiòn pùblica lo cual beneficio tanto al gobierno y a la ciudadania.

Finalmente, Damascene y Andersson (2019) reconocen que ha habido un aumento del gobierno electrónico como parte de la gestión en los gobiernos, pese que existe un sesgo en la investigación respecto a este tema, sin embargo, como resultado se obtuvo que hubo una eficiencia en la administración, los servicios públicos mejoraron considerablemente, impulso en la ética de las autoridades y un bienestar social consecuente. Asimismo, su aplicación se da porque los países desean obtener mejorar en sus relaciones internas y externas y que el sistema democrático siga escalando en sociedad. 


\section{CONCLUSIÓN O CONSIDERACIONES FINALES}

Como primera conclusión, si hablamos del área de salud, la cual ha sido la más afectada, podemos deducir lo mal preparado que se ha encontrado su equipo en la cual por priorizar la atención de los casos de contagios de COVID-19 han descuidado otras áreas en la cual en muchos hospitales más en el continente sudamericano las dejaron de lado, como las enfermedades degenerativas o la salud mental en la cual al darse cuenta de esto muchos encargados de la salud mental empezaron a realizar estrategias en la que se implementó la tecnología.

Como segunda conclusión, otra área definitivamente afectada ha sido la educación en la cual tras cerrarse millones de colegios y universidades alrededor del mundo la gente tras llevar una cuarentena obligatoria tuvo que aprender y tener clases vía online en la cual fue un reto enorme para los docentes que nunca han tocado una computadora o han tenido poco contacto con ella, tras la maravillosas estrategias del personal docente y las herramientas brindadas por los portales de videoconferencias actualmente se puede gozar de una enseñanza significativa.

Como última conclusión, la tecnología ha sido nuestra primera aliada en esta terrorífica y grave situación en la que se encuentra el mundo, siendo considerada como una de las diversas estrategias utilizadas para sobrevivir dentro de un nuevo sistema de vida. Asimismo, se precisa que al principio estas estrategias quizá no fueron de buena forma aceptadas y ciertamente complicadas para algunos, sin embargo, ahora se encuentran en gran medida implementadas y se le puede sacar partido a muchas de ellas ya que se puede aprender, trabajar e informar con una gran calidad de por medio, el ser humano siempre encontrara las formas y estrategias para poder vivir cómodo y feliz.

\section{LISTA DE REFERENCIAS}

Bansal, A., Prathap Padappayil , R., Garg, C., \& Singal, A. (2020). Utility of Artificial Intelligence Amidst the COVID 19 Pandemic: A Review. Journal of Medical Systems(156). https://link.springer.com/article/10.1007/s10916-020-01617-3

Cepeda, J., \& Cifuentes, W. (2019). Quality Management System in the Public Sector. A literary review. Podium(36), 35-54. doi:https://doi.org/10.31095/podium.2019.36.3 
Cohen , S., Mamakou, X., \& Karatzimas, S. (2017). It-enhanced popular reports: Analyzing citizen preferences. Government Information Quarterly, 34(3), 283295. doi:https://doi.org/10.1016/j.giq.2017.04.003

Damascene, J., \& Anderson, A. (2019). The public value of e-Government- A literatura review. Government Information Quarterly, 36(2), 167-178. doi:https://doi.org/10.1016/j.giq.2019.01.001

Hellewell, J., Abbott, S., Gimma , A., Bosse, N., Christopher , J., Russell, T., JKucharski, A. (2020). Feasibility of controlling COVID-19 outbreaks by isolation of cases and contacts. The Lancet Global Health, 8(4). doi:https://doi.org/10.1016/S2214109X(20)30074-7

Hodges, J., \& Howieson, B. (2017). The challenges of leadership in the third sector. European Management Journal, doi:https://doi.org/10.1016/j.emj.2016.12.006

Llerena Lanza, R., \& Sànchez Narvàes, C. (2020). Emergency, management, vulnerability and responses to the impact of the COVID-19 pandemic in Peru. Scielo. https://preprints.scielo.org/index.php/scielo/preprint/view/94/version/99

Machìn Hernàndez, M., Sànchez Vignau, B., Lòpez Rodrìgez, M., \& Puentes Alvarez, P. (2019). La gestión pública local como garante de la eficacia en la administración pública cubana. Cooperativismo y Desarrollo, 7(2). 340X2019000200212

Mauro , M., \& Giancotti, M. (2021). Italian responses to the COVID-19 emergency: Overthrowing 30 years of health reforms? Health Policy, 125(4), 548-552. doi:https://doi.org/10.1016/j.healthpol.2020.12.015

McBride, K., \& Draheim, D. (2020). On Complex Adaptive Systems and Electronic Government: A Proposed Theoretical Approach for Electronic Government Studies. The Electronic Journal of e-Government, 18(1), 43-53. doi:10.34190/EJEG.18.1.004

Morveli, G. (2021). Approaches to Public Management and its influence on the government Peruvian 1990 to 2020. Ciencia Latina Revista Científica Multidisciplinar, 5(3), 3496-3512.doi:https://doi.org/10.37811/cl_rcm.v5i3.546

Núñez Moscoso, J. (2017). Mixed methods in education research: towards a reflexive use. Cadernos de Pesquisa(47). doi:https://doi.org/10.1590/198053143763 
Oluyase , A., Hocaoglu, M., Cripps, R., Maddocks , M., \& Walshe , P. (2021). The Challenges of Caring for People Dying From COVID-19: A Multinational, Observational Study (CovPall). Journal of Pain and Symptom Management, 62(3), 460-470. doi:https://doi.org/10.1016/j.jpainsymman.2021.01.138

Ornell, F., Ferreira Moura, H., Nichterwitz Scherer, J., Pechansky, F., Felix , H., \& Diemen, L. (2020). The COVID-19 pandemic and its impact on substance use: Implications for prevention and treatment. Psychiatry Research, 289. doi:https://doi.org/10.1016/j.psychres.2020.113096

Pliscoff Varas, C. (2017). Implementing the new public management:problems and challenges to public ethics. Convergencia, 24(73). http://www.scielo.org.mx/scielo.php?pid=S1405$14352017000100141 \&$ script $=$ sci_abstract $\&$ tlng $=$ pt

Rana, N., Dwivedi, Y., Luthra, S., Lal, B., \& Alryalat, M. (2017). Advances in Electronic Government (e- Government) Adoption Research in SAARC Countries. Lecture Notes in Computer Science, 10595. doi:https://doi.org/10.1007/978-3-319-68557$1 \_14$

Sànchez Flores, F. (2019). Fundamentos epistémicos de la investigación cualitativa y cuantitativa: Consensos y disensos. Revista Digital de Investigación en Docencia Universitaria, 13(1), 102-122. doi:http://dx.doi.org/10.19083/ridu.2019.644

Snyder, H. (2019). Literature review as a research methodology: An overview and guidelines. Journal of Business Research, 104, 333-339. doi:https://doi.org/10.1016/j.jbusres.2019.07.039

Tobi, H., \& Kampen, J. (2018). Research design: the methodology for interdisciplinary research framework. Quality \& Quantity, 52, 1209-1255. doi:https://doi.org/10.1007/s11135-017-0513-8

Troy, J. (2020). E-Government in the Philippines: An Assessment. Ciencia (HEALS), 1(1), 26-47. https://ssrn.com/abstract=3762071 